\title{
On the complete arcs containing the quadrangles constructing the Fano planes of the left near field plane of order 9
}

\author{
Ayse Bayar ${ }^{\star \star}$, Ziya Akca, E. Altintas, Suheyla Ekmekci \\ Department of Mathematics-Computer, Faculty of Science and Arts, Eskisehir Osmangazi University, Eskisehir, Turkey \\ Received: 12 October 2016, Accepted: 14 November 2016 \\ Published online: 17 December 2016.
}

\begin{abstract}
In this paper, we introduce a method to find arcs in the projective plane of order 9 coordinatized by elements of a left nearfield of order 9. We give an algorithm for checking arcs in this projective plane and apply the algorithm (implemented in C\#) to determine and classify arcs.
\end{abstract}

Keywords: Near field, projective plane, arc.

\section{Introduction}

A projective plane $\pi$ consists of a set $P$ of points, and a set $L$ of subsets of $P$, called lines, such that every pair of points is contained in exactly one line, every two distinct lines intersect in exactly one point, and there exist four points in such a position that they pairwise define six distinct lines. A subplane of a projective plane $\pi$ is a set $B$ of points and lines which is itself a projective plane, relative to the incidence relation given in $\pi$.

It is well known that there exist at least four non-isomorphic projective planes of order 9. The known four distinct projective planes of order 9 are extensively studied by Room Kirkpatrick [4]. These are Desarguesian plane, the left nearfield plane, the right nearfield plane and Hughes plane. The last three planes of order 9 are called "miniquaternion planes" because they can be coordinatized by the miniquaternion near field. O. Veblen and J. M. Wedderburn [6] discovered these miniquaternion planes in 1907.

A $(k, 2)-\operatorname{arc} K$ is a set of $k$ points no 3 of which are collinear in the plane. A $(k, 2)-\operatorname{arc}$ is called simply an arc of size $k$ or a $k$ arc. $\mathrm{A}(k, 3)-\operatorname{arc} K$ is a set of $k$ points no 4 of which are collinear of this plane.

In [1,5], an algorithm (implemented in C\#) to determine and classify Fano subplanes of the projective plane of order 9 coordinatized by elements of a left nearfield of order 9 is given and 18 complete quadrangles which generate Fano plane are determined. In this paper, the projective plane whose algebraic structure is the left nearfield plane of order 9 is constructed by using homogene coordinates and then all complete arcs containing the Fano plane are obtained by using "completion procedure" in this projective plane. We give an algorithm for checking arcs in this projective plane and apply the algorithm (implemented in $\mathrm{C \# )}$ to determine and classify arcs.

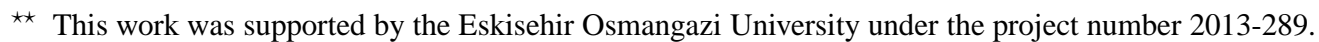




\section{The left nearfield system of order 9}

The regular near field of order $q^{2}$, for $q$ an odd prime power, are defined taking the elements of $G F\left(q^{2}\right)$, using the field addition and definition a new multiplication on the elements in terms of the field multiplication. This gives an algebraic system in which the non-zero elements form a group under the multiplication and the right or left distributive laws hold. The near field can be used to define and coordinatize the near field plane of order 9 .

We give the left near field of order 9 by taking the elements of $G F(3)$ and using the field addition and a new multiplication on the elements in terms of the left near field multiplication, $[1,2,3,5]$.

Definition 1. A left nearfield is a system $(S, \oplus, \odot)$, where $\oplus$ and $\odot$ are binary operations on the set $S$ and

(i) $S$ is finite

(ii) $(S, \oplus)$ is a group, with identity 0

(iii) $(S \backslash\{0\}, \odot)$ is a group, with identity 1

(iv) $0 \odot x=0$ for all $x \in S$

(v) $\odot$ is left distributive over $\oplus$, that is $x \odot(y \oplus z)=(x \odot y) \oplus(x \odot z)$ for all $x, y, z \in S$

(vi) Given $m, n, k \in S$ with $m \neq n$, there exists a unique $x \in S$ such that $-m \odot x \oplus n \odot x=k$.

Let $\left(F_{3},+,.\right)$ be the Galois field of order 3. We now construct $(S, \oplus, \odot)$, using $F_{3}$, a left nearfield of order 9 .

The nine elements of $S$ are $a+\lambda b, a, b \in F_{3}, \lambda \notin F_{3}$. Addition in $S$ is defined by the rule

$$
(a+\lambda b) \oplus(c+\lambda d)=(a+c)+\lambda(b+d)
$$

and multiplication by

$$
(a+\lambda b) \odot(c+\lambda d)= \begin{cases}a c+\lambda(a d), & \text { if } \quad b=0 \\ a c-b^{-1} d f(a)+\lambda(b c-(a-1) d), & \text { if } \quad b \neq 0\end{cases}
$$

where, $a, b, c, d \in F_{3}, \lambda \notin F_{3}$ and $f(t)=t^{2}+1$ is a irreducible polynom on $F_{3}$.

For the sake of sorthness if we use $a b$ instead of $a+\lambda b$ in equation (1) and (2), then addition and multiplication tables are obtained as follows:

Table 1

\begin{tabular}{|l|l|l|l|l|l|l|l|l|l|}
\hline$\oplus$ & 00 & 01 & 02 & 10 & 11 & 12 & 20 & 21 & 22 \\
\hline 00 & 00 & 01 & 02 & 10 & 11 & 12 & 20 & 21 & 22 \\
\hline 01 & 01 & 02 & 00 & 11 & 12 & 10 & 21 & 22 & 20 \\
\hline 02 & 02 & 00 & 01 & 12 & 10 & 11 & 22 & 20 & 21 \\
\hline 10 & 10 & 11 & 12 & 20 & 21 & 22 & 00 & 01 & 02 \\
\hline 11 & 11 & 12 & 10 & 21 & 22 & 20 & 01 & 02 & 00 \\
\hline 12 & 12 & 10 & 11 & 22 & 20 & 21 & 02 & 00 & 01 \\
\hline 20 & 20 & 21 & 22 & 00 & 01 & 02 & 10 & 11 & 12 \\
\hline 21 & 21 & 22 & 20 & 01 & 02 & 00 & 11 & 12 & 10 \\
\hline 22 & 22 & 20 & 21 & 02 & 00 & 01 & 12 & 10 & 11 \\
\hline
\end{tabular}


Table 2

\begin{tabular}{|c|c|c|c|c|c|c|c|c|c|}
\hline$\odot$ & 00 & 01 & 02 & 10 & 11 & 12 & 20 & 21 & 22 \\
\hline 00 & 00 & 00 & 00 & 00 & 00 & 00 & 00 & 00 & 00 \\
\hline 01 & 00 & 20 & 10 & 01 & 21 & 11 & 02 & 22 & 12 \\
\hline 02 & 00 & 10 & 20 & 02 & 12 & 22 & 01 & 11 & 21 \\
\hline 10 & 00 & 01 & 02 & 10 & 11 & 12 & 20 & 21 & 22 \\
\hline 11 & 00 & 12 & 21 & 11 & 20 & 02 & 22 & 01 & 10 \\
\hline 12 & 00 & 22 & 11 & 12 & 01 & 20 & 21 & 10 & 02 \\
\hline 20 & 00 & 02 & 01 & 20 & 22 & 21 & 10 & 12 & 11 \\
\hline 21 & 00 & 11 & 22 & 21 & 02 & 10 & 12 & 20 & 01 \\
\hline 22 & 00 & 21 & 12 & 22 & 10 & 01 & 11 & 02 & 20 \\
\hline
\end{tabular}

If we use the following equalities

$$
\begin{aligned}
& 0=(0,0) \\
& 1=(1,0) \\
& 2=(2,0) \\
& 3=(0,1) \\
& 4=(1,1) \\
& 5=(2,1) \\
& 6=(0,2) \\
& 7=(1,2) \\
& 8=(2,2)
\end{aligned}
$$

the addition and multiplication tables in $(S, \oplus, \odot)$ can be arranged as follows :

Table 3

\begin{tabular}{|l|l|l|l|l|l|l|l|l|l|}
\hline$\oplus$ & 0 & 1 & 2 & 3 & 4 & 5 & 6 & 7 & 8 \\
\hline 0 & 0 & 1 & 2 & 3 & 4 & 5 & 6 & 7 & 8 \\
\hline 1 & 1 & 2 & 0 & 4 & 5 & 3 & 7 & 8 & 3 \\
\hline 2 & 2 & 0 & 1 & 5 & 3 & 4 & 8 & 6 & 7 \\
\hline 3 & 3 & 4 & 5 & 6 & 7 & 8 & 0 & 1 & 2 \\
\hline 4 & 4 & 5 & 3 & 7 & 8 & 6 & 1 & 2 & 0 \\
\hline 5 & 5 & 3 & 4 & 8 & 6 & 7 & 2 & 0 & 1 \\
\hline 6 & 6 & 7 & 8 & 0 & 1 & 2 & 3 & 4 & 5 \\
\hline 7 & 7 & 8 & 6 & 1 & 2 & 0 & 4 & 5 & 3 \\
\hline 8 & 8 & 6 & 7 & 2 & 0 & 1 & 5 & 3 & 4 \\
\hline
\end{tabular}

\begin{tabular}{|l|l|l|l|l|l|l|l|l|l|}
\hline$\odot$ & 0 & 1 & 2 & 3 & 4 & 5 & 6 & 7 & 8 \\
\hline 0 & 0 & 0 & 0 & 0 & 0 & 0 & 0 & 0 & 0 \\
\hline 1 & 0 & 1 & 2 & 3 & 4 & 5 & 6 & 7 & 8 \\
\hline 2 & 0 & 2 & 1 & 6 & 8 & 7 & 3 & 5 & 4 \\
\hline 3 & 0 & 3 & 6 & 2 & 5 & 8 & 1 & 4 & 7 \\
\hline 4 & 0 & 4 & 8 & 7 & 2 & 3 & 5 & 6 & 1 \\
\hline 5 & 0 & 5 & 7 & 4 & 6 & 2 & 8 & 1 & 3 \\
\hline 6 & 0 & 6 & 3 & 1 & 7 & 4 & 2 & 8 & 5 \\
\hline 7 & 0 & 7 & 5 & 8 & 3 & 1 & 4 & 2 & 6 \\
\hline 8 & 0 & 8 & 4 & 5 & 1 & 6 & 7 & 3 & 2 \\
\hline
\end{tabular}

The system $(S, \oplus, \odot)$ satisfies the conditions of Definition 2.1 and therefore a left nearfield of order 9 .

\section{The projective plane $P_{2} S$}

It is well known that every projective plane has also an algebraic structure obtained by coordinazation. Conversely, certain algebraic structures can be used to construct projective planes.

In this section, we will construct projective plane order 9. The projective plane whose the points and the lines are coordinated by the elements of $(S, \oplus, \odot)$. 
The 91 points of $P_{2} S$ are the elements of the set

$$
\{(x, y): x, y \in S\} \cup\{(m): m \in S\} \cup\{(\infty)\} .
$$

The points of the form $(x, y)$ are called proper points, and the unique point $(\infty)$ and the points of the form $(m)$ are calledideal points. The 91 lines of $P_{2} S$ are defined to be set of points satisfying one of the three conditions:

$$
\begin{aligned}
& {[m, k]=\left\{(x, y) \in S^{2}, y=m \odot x \oplus k\right\} \cup\{(m)\}} \\
& {[a]=\left\{(x, y) \in S^{2}, x=a\right\} \cup\{(\infty)\}} \\
& {[\infty]=\{(m) \in S\} \cup\{(\infty)\} .}
\end{aligned}
$$

The 81 lines having form $y=m \odot x \oplus k$ and 9 lines having equation of the form $x=\lambda$ are called the proper lines and the unique line $[\infty]$ is called the ideal line.

The system of points, lines and incidence relation given above defines a projective plane of order 9 , which is the left nearfiled plane.

Now, we are considering the projective plane of order 9 homogeneous coordinatized by elements of the above left nearfield. We notice that the homogeneous coordinates of a point are not unique. Two triples that are multiples of each other specify the same point. Thus the same point has many sets of homogeneous coordinates: $(x, y, z)$ and $\left(x^{\prime}, y^{\prime}, z^{\prime}\right)$ represent the same point if and only if there is some $\lambda \neq 0, \lambda \in S$ such that $x^{\prime}=\lambda \odot x, y^{\prime}=\lambda \odot y, z^{\prime}=\lambda \odot z$. We convert a point expressed in Cartesian coordinates to homogeneous coordinates in left nearfield plane of order 9. We have seen that a point $(x, y)$ in the $P_{2} S$ has homogeneous coordinates $\lambda \odot(x, y, 1)=(\lambda \odot x, \lambda \odot y, \lambda \odot 1), \lambda \neq 0, \lambda \in S$. Homogeneous coordinates of the form $\lambda \odot(m, 1,0)$ do correspond to all ideal points $(m), m, \lambda \in S^{*}$ in the $P_{2} S$. Homogeneous coordinates of the form $(\lambda, 0,0)$ do correspond to the unique point at infinity in the $P_{2} S$. We have seen that a line $[m, k]$ in the $P_{2} S$ has homogeneous coordinates $\mu \odot[m,-1, k]=[\mu \odot m, \mu \odot(-1), \mu \odot k], \mu \neq 0, \mu \in S$. Homogeneous coordinates of the form $\mu \odot[x, 0,1]$ do correspond to all lines $[a], a \neq 0, a \in S$ in the $P_{2} S$. Homogeneous coordinates of the form $[0,0, \mu]$ do correspond to the unique line $[\infty]$ at infinity in the $P_{2} S$.

A line in the $P_{2} S$ has general equation $y=m \odot x \oplus k$. Suppose $\left(x_{1}, x_{2}, x_{3}\right), x_{3} \neq 0$ are the homogeneous coordinates of a point $(x, y)$ on the line; hence $x=x_{3}^{-1} \odot x_{1}$ and $y=x_{3}^{-1} \odot x_{2}$. Substituting for $x$ and $y$ in the line equation and multiplying through by $x_{3}$, yields the conditions for $\left(x_{1}, x_{2}, x_{3}\right)$ to be the homogeneous coordinates of a point on the line:

$$
m \odot x_{1} \oplus(-1) \odot x_{2} \oplus k \odot x_{3}=0 .
$$

The following table lists all homogeneous coordinates of the 91 points and lines in the projective plane of order 9 coordinatized by elements of the above left nearfield.

\section{The complete arcs containing the quadrangles constructing the Fano planes in the projective plane $P_{2} S$}

A $(k, n)-\operatorname{arc} K$ in a projective plane is a set of $k$ points, such that some $n$, but no $n+1$ of them are collinear. A $(k, n)-\operatorname{arc}$ $K$ is complete if it is not contained in a $(k+1, n)-$ arc. A line of the projective plane containing exactly $i$ points of a $(k, n)-\operatorname{arc}$ is called an $i$-secant of $K$. For a $(k, 3)-\operatorname{arc}$ each line of projective plane is a 3-secant, 2-secant, 1-secant, or 0 -secant.

It is known that a finite projective plane is coordinatized by any quadrangle $O, I, X, P$ (4 points, no 3 on a line). In [1,5], It 


\begin{tabular}{|c|c|c|c|c|c|c|c|c|c|c|}
\hline P) & & & & $b$ & & & & & & \\
\hline I $(1,0,0)$ & 2 & 21 & 20 & 29 & 38 & 47 & 56 & 65 & 74 & 83 \\
\hline $2(0,1,0)$ & 1 & 11 & 12 & 13 & 14 & 15 & 16 & 17 & 18 & 19 \\
\hline $3(1,1,0)$ & 4 & 11 & 22 & 30 & 44 & 55 & 63 & 68 & 79 & 87 \\
\hline $4(2,1,0)$ & 3 & 11 & 21 & 31 & 41 & S1 & 61 & 71 & 81 & 91 \\
\hline $5(3,1,0)$ & 5 & 11 & 23 & 35 & 40 & 54 & 60 & 66 & 82 & 88 \\
\hline $6(4,1,0)$ & 6 & 11 & 24 & 37 & 43 & 49 & 62 & 72 & $\pi$ & 84 \\
\hline $7(5,1,0)$ & 7 & 11 & 25 & 36 & 46 & so & 58 & 69 & 75 & B9 \\
\hline s $(6,1,0)$ & 8 & 11 & 26 & 32 & 39 & 52 & 64 & 67 & 78 & 90 \\
\hline $9(7,1,0)$ & 9 & 11 & 27 & 34 & 42 & 53 & 57 & 73 & 76 & B6 \\
\hline $10(8,1,0)$ & 10 & 11 & 28 & 33 & 45 & 48 & 59 & 70 & 80 & BS \\
\hline $11(0,0,1)$ & 1 & 2 & 3 & 4 & 5 & 6 & 7 & 8 & 9 & 10 \\
\hline $12(1,0,1)$ & 2 & 13 & 22 & 31 & 40 & 49 & 58 & 67 & 76 & B5 \\
\hline $13(2,0,1)$ & 2 & 12 & 21 & 30 & 39 & 48 & 57 & 66 & 73 & 84 \\
\hline $24(3,0,1)$ & 2 & 14 & 23 & 32 & 41 & so & 59 & 68 & $\pi$ & B6 \\
\hline $15(4,0,1)$ & 2 & 15 & 24 & 33 & 42 & 51 & 60 & 69 & 78 & 87 \\
\hline $10(5,0,1)$ & 2 & 10 & 25 & 34 & 43 & $\$ 2$ & 01 & 70 & 79 & 88 \\
\hline $17(6,0,1)$ & 2 & 17 & 26 & 35 & 44 & 53 & 62 & 71 & 80 & B9 \\
\hline $18(7,0,1)$ & 2 & 18 & 27 & 36 & 45 & 54 & 63 & 72 & 81 & 90 \\
\hline $19(8,0,1)$ & 2 & 19 & 28 & 37 & 46 & 55 & 64 & 73 & 82 & 91 \\
\hline $20(0,1,1)$ & 1 & 29 & 30 & 31 & 32 & 33 & 34 & 35 & 36 & 37 \\
\hline $21(1,1,1)$ & 4 & 13 & 21 & 29 & 46 & 54 & 62 & 70 & 78 & 86 \\
\hline $22(2,1,1)$ & 3. & 12 & 22 & 29 & 42 & 52 & 59 & 72 & 82 & 89 \\
\hline $23(3,1,1)$ & 5 & 14 & 26 & 29 & 45 & 51 & 58 & 73 & 79 & 84 \\
\hline $24(4,1,1)$ & 6 & 15 & 28 & 29 & 40 & 53 & 61 & 68 & 75 & 90 \\
\hline $25(5,1,1)$ & 7 & 16 & 27 & 29 & 41 & 49 & 64 & 66 & 80 & 87 \\
\hline $26(6,1,1)$ & 8 & 17 & 23 & 29 & 43 & 55 & 57 & 69 & 81 & 85 \\
\hline $27(7,1,1)$ & 9 & 18 & 25 & 29 & 44 & 48 & 60 & 67 & $n$ & 91 \\
\hline $28(8,1,1)$ & 10 & 15 & 24 & 29 & 39 & 30 & 63 & 71 & 76 & 83 \\
\hline $29(0,2,1)$ & 1 & 20 & 21 & 22 & 23 & 24 & 25 & 26 & 27 & 28 \\
\hline $30(1,2,1)$ & 3 & 13 & 20 & 30 & 43 & so & 60 & 73 & 80 & 90 \\
\hline $31(2,2,1)$ & 4 & 12 & 20 & 31 & 45 & 53 & 64 & 69 & 77 & 88 \\
\hline $32(3,2,1)$ & 8 & 14 & 20 & 35 & 46 & 48 & 61 & $n$ & 76 & 87 \\
\hline $33(4,2,1)$ & 10 & 15 & 20 & 37 & 41 & 54 & 57 & 67 & 79 & 89 \\
\hline $34(5,2,1)$ & 9 & 16 & 20 & 36 & 39 & 51 & 62 & 68 & 82 & 85 \\
\hline $35(6,2,1)$ & 5 & 17 & 20 & 32 & 42 & 49 & 63 & 70 & 75 & 91 \\
\hline $36(7,2,1)$ & 7 & 18 & 20 & 34 & 40 & 55 & 59 & $7 t$ & 78 & 84 \\
\hline $37(8,2,1)$ & 6 & 19 & 20 & 33 & 44 & 52 & 58 & 66 & 81 & 86 \\
\hline $33(0,3,1)$ & 1 & 38 & 39 & 40 & 41 & 42 & 43 & 44 & 45 & 46 \\
\hline $39(1,3,1)$ & 8 & 13 & 28 & 34 & 38 & 51 & 63 & 66 & 77 & 89 \\
\hline $40(2,3,1)$ & 5 & 12 & 24 & 36 & 38 & 55 & 61 & 67 & 80 & 86 \\
\hline $41(3,3,1)$ & 4 & 14 & 27 & 37 & 38 & 52 & 60 & $\pi$ & 75 & 85 \\
\hline $42(4,3,1)$ & 7 & 15 & 22 & 32 & 38 & 48 & 62 & 73 & 81 & 38 \\
\hline $43(5,3,1)$ & 10 & 16 & 23 & 30 & 38 & 53 & 58 & 72 & 78 & 91 \\
\hline $44(6,3,1)$ & 3 & 17 & 25 & 33 & 38 & 54 & 64 & 68 & 76 & 84 \\
\hline $45(7,3,1)$ & 6 & 18 & 26 & 31 & 38 & so & 57 & 70 & 82 & 87 \\
\hline $45(8,3,1)$ & 9 & 19 & 21 & 35. & 38 & A9 & 59 & 69 & 79 & 90 \\
\hline $47(0,4,1)$ & 1 & 47 & 48 & 49 & So & 51 & 52 & 53 & 54 & 55 \\
\hline
\end{tabular}

\begin{tabular}{|c|c|c|c|c|c|c|c|c|c|c|}
\hline $48(1,4,1)$ & 10 & 13 & 27 & 32 & 44 & 47 & 61 & 69 & 82 & 84 \\
\hline $49(2,4,1)$ & 6 & 12 & 25 & 35 & 41 & 47 & 63 & 73 & 78 & 85 \\
\hline $50(3,4,1)$ & 9 & 14 & 24 & 30 & 40 & 47 & 64 & 70 & 81 & 89 \\
\hline $51(4,4,1)$ & 4 & 15 & 26 & 36 & 43 & 47 & 59 & 66 & 76 & 91 \\
\hline $52(5,4,1)$ & 5 & 16 & 22 & 33 & 46 & 47 & 57 & 71 & 77 & 90 \\
\hline $53(6,4,1)$ & 7 & 17 & 28 & 31 & 39 & 47 & 60 & 72 & 79 & 86 \\
\hline $54(7,4,1)$ & 8 & 18 & 21 & 37 & 42 & 47 & 58 & 68 & 80 & 88 \\
\hline $55(8,4,1)$ & 3 & 19 & 23 & 34 & 45 & 47 & 62 & 67 & 75 & 87 \\
\hline $56(0,5,1)$ & 1 & 56 & 57 & 58 & 59 & 60 & 61 & 62 & 63 & 64 \\
\hline $57(1,5,1)$ & 9 & 13 & 26 & 33 & 41 & 55 & 56 & 72 & 75 & 88 \\
\hline $58(2,5,1)$ & 7 & 12 & 23 & 37 & 44 & 51 & 56 & 70 & 76 & 90 \\
\hline $59(3,5,1)$ & 6 & 14 & 22 & 34 & 39 & 54 & 56 & 69 & 80 & 91 \\
\hline $60(4,5,1)$ & 8 & 15 & 25 & 30 & 45 & 49 & 56 & $\pi$ & 82 & 86 \\
\hline $61(5,5,1)$ & 4 & 16 & 28 & 35 & 42 & 50 & 56 & 67 & 81 & 94 \\
\hline $62(6,5,1)$ & 10 & 17 & 21 & 35 & 40 & 52 & 56 & 73 & 77 & 87 \\
\hline $63(7,5,1)$ & 3 & 18 & 24 & 32 & 46 & 53 & 56 & 66 & 79 & 85 \\
\hline $64(8,5,1)$ & 5 & 19 & 27 & 31 & 43 & 48 & 56 & 68 & 78 & 89 \\
\hline $65(0,6,1)$ & 1 & 65 & 66 & 67 & 68 & 69 & 70 & 71 & 72 & 73 \\
\hline $66(1,6,1)$ & 5 & 13 & 25 & 37 & 39 & 53 & 59 & 65 & 81 & 87 \\
\hline $67(2,6,1)$ & 8 & 12 & 27 & 33 & 40 & 50 & 62 & 65 & 79 & 91 \\
\hline $68(3,6,1)$ & 3 & 14 & 28 & 36 & 44 & 49 & 57 & 65 & 78 & 88 \\
\hline $69(4,6,1)$ & 9 & 15 & 23 & 31 & 46 & 52 & 63 & 65 & 80 & 84 \\
\hline $70(5,6,1)$ & 6 & 16 & 21 & 32 & 45 & 55 & 60 & 65 & 76 & 89 \\
\hline $7(6,6,1)$ & 4 & 17 & 24 & 34 & 41 & 48 & 58 & 65 & 82 & 90 \\
\hline $72(7,6,1)$ & 10 & 18 & 22 & 35 & 43 & 51 & 64 & 65 & 75 & 86 \\
\hline $73(8,6,1)$ & 7 & 19 & 26 & 30 & 42 & 54 & 61 & 65 & 77 & 85 \\
\hline $74(0,7,1)$ & 1 & 74 & 75 & 75 & 77 & 78 & 79 & 80 & 81 & 82 \\
\hline $75(1,7,1)$ & 7 & 13 & 24 & 35 & 45 & 52 & 57 & 68 & 74 & 91 \\
\hline $76 \cdot(2,7,1)$ & 9 & 12 & 28 & 32 & 43 & 54 & 58 & 71 & 74 & 87 \\
\hline $77(3,7,1)$ & 10 & 14 & 25 & 31 & 42 & 55 & 62 & 66 & 74 & 90 \\
\hline $78(4,7,1)$ & 5 & 15 & 21 & 34 & 44 & 50 & 64 & 72 & 74 & 85 \\
\hline $79(5,7,1)$ & 3 & 16 & 26 & 37 & 40 & 48 & 63 & 69 & 74 & 86 \\
\hline $80(6,7,1)$ & 6 & 17 & 27 & 30 & 46 & 51 & 59 & 67 & 74 & 88 \\
\hline $81 .(7,7,1)$ & 4 & $1 B$ & 23 & 33 & 39 & 49 & 61 & 73 & 74 & 89 \\
\hline $82(8,7,1)$ & 8 & 19 & 22 & 36 & 41 & 53 & 60 & 70 & 74 & 84 \\
\hline $83(0,8,1)$ & 1 & 83 & 84 & 85 & 86 & 87 & 88 & 89 & 90 & 91 \\
\hline $84(1,8,1)$ & 6 & 13 & 23 & 36 & 42 & 48 & 64 & 71 & 79 & 83 \\
\hline $85(2,8,1)$ & 10 & 12 & 26 & 34 & 46 & 49 & 60 & 68 & 81 & 83 \\
\hline $86(3,8,1)$ & 7 & 14 & 21 & 33 & 43 & 53 & 63 & 67 & 82 & 83 \\
\hline $87(4,8,1)$ & 3 & 15 & 27 & 35 & 39 & 55 & 58 & 70 & 77 & 83 \\
\hline $88(5,8,1)$ & B & 16 & 24 & 31 & 44 & 54 & 59 & 73 & 75 & 83 \\
\hline $89(6,8,1)$ & 9 & 17 & 22 & 37 & 45 & 50 & 61 & 66 & 78 & 83 \\
\hline $90(7,8,1)$ & 5 & 18 & 28 & 30 & 41 & 52 & 62 & 69 & 76 & 83 \\
\hline $91(8,8,1)$ & 4 & 19 & 25 & 32 & 40 & 51. & 57 & 72 & 80 & 83 \\
\hline
\end{tabular}

is seen that there are 18 Fano subplanes containing $I=(1,1,1), X=(1,0,0), O=(0,0,1), P=(a, b, 1)$ with $a, b \in F_{3}$ in $P_{2} S$.

In this section, $(k, 2)$-arcs containing Fano planes of $P_{2} S$ are determined selecting the quadrangle $O, I, X, P$ such that $I=(1,1,1), X=(1,0,0), O=(0,0,1), P=(a, b, 1)$ with $a, b \in F_{3}$ in $P_{2} S$ and the algorithm used in the classification of $(k, 2)$-arcs containing Fano planes of $P_{2} S$ is described. By applying this algorithm (implemented $\left.C \#\right)$ a full listing the complete $(k, 2)$-arcs containing Fano planes of $P_{2} S$ has been achieved.

\subsection{Method and algorithm}

Our method had been staged into 2 steps. Firstly, we consider a quadrangle from Fano subplanes containing $I=(1,1,1)$, $X=(1,0,0), O=(0,0,1), P=(a, b, 1)$ with $a, b \in F_{3}$ in $P_{2} S$. Secondly, a point not on the lines of selected Fano plane is 
added the set $\{O, I, X, P\}$ and then a new point not in spanned by the previous set. It is iterated this argument. The process ends when a complete arc is obtained. Now, we give an algorithm (implemented $C \#$ ) to find these complete arcs.

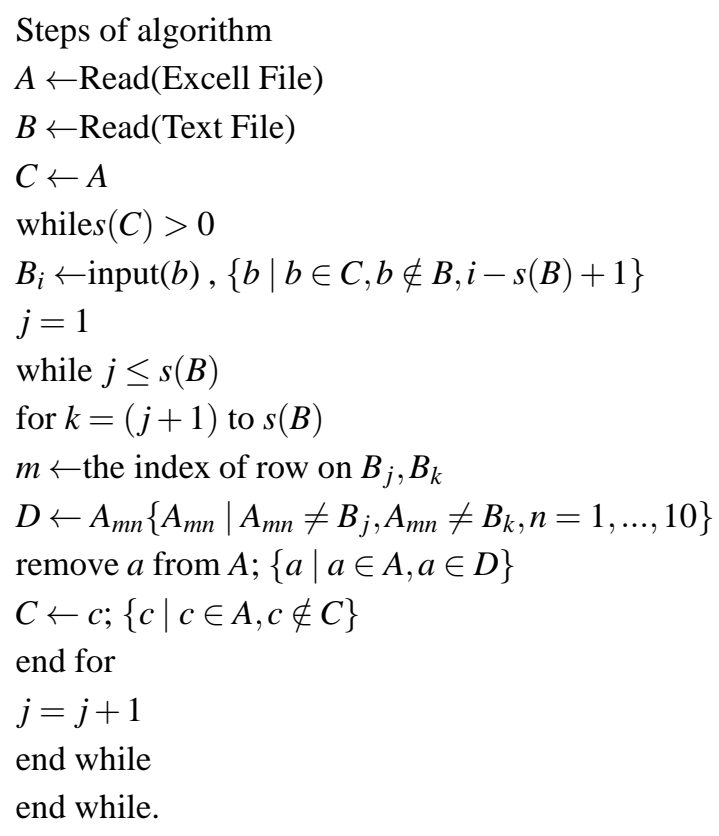

\subsection{List of the complete arcs containing the quadrangles constructing the Fano planes}

It is determined the complete $(k, 2)$ - arcs containing the quadrangles constructing the Fano subplanes in $P_{2} S$ by using a computer programme in [7].

$(6,2)-\operatorname{arc}$ is $\{11,6,11,21,38,58\}$

$(7,2)$ - arcs are

$$
\begin{aligned}
& \{1,4,11,21,35,38,88\},\{1,4,11,21,37,38,73\},\{1,4,11,21,37,38,77\} \\
& \{1,4,11,21,38,53,84\},\{1,4,11,21,38,60,66\},\{1,4,11,21,38,66,88\} \\
& \{1,4,11,21,38,72,84\},\{1,5,11,21,38,53,89\},\{1,5,11,21,38,54,87\} \\
& \{1,5,11,21,38,57,70\},\{1,5,11,21,38,57,72\},\{1,6,11,21,38,57,68\} \\
& \{1,6,11,21,38,57,73\},\{1,8,11,21,30,53,80\},\{1,11,21,33,38,53,64\} \\
& \{1,8,11,21,38,57,72\},\{1,9,11,21,30,38,70\},\{1,9,11,21,30,38,78\} \\
& \{1,9,11,21,36,38,66\},\{1,9,11,21,36,38,87\},\{1,9,11,21,38,55,86\} \\
& \{1,9,11,21,38,62,66\},\{1,9,11,21,38,68,86\},\{1,10,11,21,30,38,73\} \\
& \{1,10,11,21,30,38,90\},\{1,10,11,21,34,38,84\},\{1,10,11,21,38,57,68\} \\
& \{1,10,11,21,38,57,73\},\{1,10,11,21,38,59,68\},\{1,10,11,21,38,64,73\} \\
& \{1,10,11,21,38,68,84\},\{1,11,21,30,38,49,73\},\{1,11,21,30,38,58,70\} \\
& \{1,11,21,33,38,53,70\},\{1,11,21,33,38,54,68\},\{1,11,21,33,38,64,79\} \\
& \{1,11,21,33,38,67,89\},\{1,11,21,33,38,79,86\},\{1,11,21,34,38,49,60\} \\
& \{1,11,21,34,38,49,86\},\{1,11,21,34,38,60,68\},\{1,11,21,34,38,72,78\} \\
& \{1,11,21,34,38,62,73\},\{1,11,21,34,38,82,86\},\{1,11,21,35,38,49,67\} \\
& \{1,11,21,35,38,49,72\},\{1,11,21,35,38,55,76\},\{1,11,21,35,38,59,68\} \\
& \{1,11,21,35,38,59,72\},\{1,11,21,35,38,60,86\},\{1,11,21,36,38,54,59\} \\
& \{1,11,21,36,38,55,57\},\{1,11,21,36,38,55,76\},\{1,11,21,36,38,55,88\}
\end{aligned}
$$


$\{1,11,21,36,38,59,82\},\{1,11,21,36,38,60,77\},\{1,11,21,36,38,77,88\}$ $\{1,11,21,37,38,50,90\},\{1,11,21,37,38,54,57\},\{1,11,21,37,38,54,89\}$ $\{1,11,21,37,38,55,62\},\{1,11,21,37,38,62,76\},\{1,11,21,37,38,62,87\}$ $\{1,11,21,37,38,76,90\},\{1,11,21,38,49,58,72\},\{1,11,21,38,49,60,86\}$ $\{1,11,21,38,49,62,66\},\{1,11,21,38,49,62,73\},\{1,11,21,38,49,72,78\}$ $\{1,11,21,38,49,73,79\},\{1,11,21,38,49,77,87\},\{1,11,21,38,49,77,88\}$ $\{1,11,21,38,50,64,88\},\{1,11,21,38,53,64,70\},\{1,11,21,38,53,70,76\}$ $\{1,11,21,38,53,70,77\},\{1,11,21,38,53,70,90\},\{1,11,21,38,54,59,89\}$ $\{1,11,21,38,54,59,79\},\{1,11,21,38,54,64,79\},\{1,11,21,38,54,68,76\}$ $\{1,11,21,38,55,59,72\},\{1,11,21,38,55,62,88\},\{1,11,21,38,55,72,78\}$ $\{1,11,21,38,57,77,88\},\{1,11,21,38,57,79,86\},\{1,11,21,38,58,70,76\}$ $\{1,11,21,38,58,72,84\},\{1,11,21,38,59,72,78\},\{1,11,21,38,59,78,89\}$ $\{1,11,21,38,60,66,76\},\{1,11,21,38,60,68,76\},\{1,11,21,38,60,68,90\}$ $\{1,11,21,38,60,77,86\},\{1,11,21,38,62,73,90\},\{1,11,21,38,62,76,90\}$ $\{1,11,21,38,64,73,87\},\{1,11,21,38,72,78,89\},\{1,11,21,38,73,79,89\}$ $\{1,11,21,38,64,70,77\},\{1,11,21,38,64,79,88\},\{1,11,21,38,64,70,87\}$ $\{1,11,21,38,64,70,77\},\{1,11,21,38,64,79,88\},\{1,11,21,38,64,70,87\}$ $\{1,11,21,38,67,76,87\},\{1,11,21,38,68,76,84\},\{1,11,21,38,70,76,87\}$

$(8,2)-\operatorname{arcs}$ are

$\{1,4,11,21,34,38,48,64\},\{1,4,11,21,34,38,60,73\},\{1,4,11,21,34,38,64,73\}$ $\{1,4,11,21,34,38,64,84\},\{1,4,11,21,34,38,75,88\},\{1,4,11,21,35,38,48,60\}$ $\{1,4,11,21,35,38,53,76\},\{1,4,11,21,35,38,72,89\},\{1,4,11,21,36,38,48,59\}$ $\{1,4,11,21,36,38,66,77\},\{1,4,11,21,36,38,59,72\},\{1,4,11,21,36,38,72,88\}$ $\{1,4,11,21,36,38,59,77\},\{1,4,11,21,37,38,64,67\},\{1,4,11,21,37,38,64,75\}$ $\{1,4,11,21,37,38,67,76\},\{1,4,11,21,37,38,67,89\},\{1,4,11,21,38,48,67,76\}$ $\{1,4,11,21,38,50,59,72\},\{1,4,11,21,38,50,60,77\},\{1,4,11,21,38,50,64,73\}$ $\{1,4,11,21,38,50,64,75\},\{1,4,11,21,38,50,64,84\},\{1,4,11,21,38,50,72,75\}$ $\{1,4,11,21,38,50,72,88\},\{1,4,11,21,38,53,67,76\},\{1,4,11,21,38,53,67,89\}$ $\{1,4,11,21,38,58,66,76\},\{1,4,11,21,38,58,66,77\},\{1,4,11,21,38,58,67,84\}$ $\{1,4,11,21,38,58,67,89\},\{1,4,11,21,38,58,77,89\},\{1,4,11,21,38,59,77,89\}$ $\{1,4,11,21,38,64,67,84\},\{1,5,11,21,38,48,70,87\},\{1,6,11,21,33,38,50,78\}$ $\{1,4,11,21,38,66,77,89\},\{1,4,11,21,38,72,75,88\},\{1,5,11,21,30,38,49,58\}$ $\{1,5,11,21,30,38,49,60\},\{1,5,11,21,30,38,49,77\},\{1,5,11,21,30,38,54,58\}$ $\{1,5,11,21,30,38,54,89\},\{1,5,11,21,30,38,58,89\},\{1,5,11,21,30,38,60,77\}$ $\{1,5,11,21,34,38,49,58\},\{1,5,11,21,34,38,54,58\},\{1,5,11,21,34,38,54,72\}$ $\{1,5,11,21,34,38,58,70\},\{1,5,11,21,34,38,68,75\},\{1,5,11,21,34,38,72,82\}$ $\{1,5,11,21,34,38,72,84\},\{1,5,11,21,37,38,54,75\},\{1,5,11,21,37,38,55,57\}$ $\{1,5,11,21,37,38,55,86\},\{1,5,11,21,37,38,57,87\},\{1,5,11,21,37,38,82,86\}$ $\{1,5,11,21,38,48,82,89\},\{1,5,11,21,38,49,58,77\},\{1,5,11,21,38,49,77,86\}$ $\{1,5,11,21,38,53,57,77\},\{1,5,11,21,38,53,68,77\},\{1,5,11,21,38,53,68,84\}$ $\{1,5,11,21,38,54,58,72\},\{1,5,11,21,38,54,72,89,\{1,5,11,21,38,55,57,86\}$ $\{1,5,11,21,38,55,72,82\},\{1,5,11,21,38,55,82,86\},\{1,5,11,21,38,57,77,86\}$ $\{1,5,11,21,38,58,70,77\},\{1,5,11,21,38,58,72,89\},\{1,5,11,21,38,60,68,77\}$ $\{1,5,11,21,38,60,68,84\},\{1,5,11,21,38,60,77,87\},\{1,5,11,21,38,68,82,86\}$ 
$\{1,5,11,21,38,72,82,84\},\{1,6,11,21,33,38,50,60\},\{1,6,11,21,33,38,50,68\}$ $\{1,6,11,21,33,38,53,68\},\{1,6,11,21,33,38,75,87\},\{1,6,11,21,35,38,48,79\}$ $\{1,6,11,21,35,38,48,89\},\{1,6,11,21,35,38,53,88\},\{1,6,11,21,35,38,57,79\}$ $\{1,6,11,21,35,38,62,75\},\{1,6,11,21,35,38,62,88\},\{1,6,11,21,35,38,79,88\}$ $\{1,6,11,21,36,38,54,57\},\{1,6,11,21,36,38,54,87\},\{1,6,11,21,36,38,57,88\}$ $\{1,6,11,21,36,38,60,66\},\{1,6,11,21,36,38,60,78\},\{1,6,11,21,36,38,60,87\}$ $\{1,6,11,21,36,38,78,87\},\{1,6,11,21,38,48,60,73\},\{1,6,11,21,38,48,60,87\}$ $\{1,6,11,21,38,48,73,79\},\{1,6,11,21,38,50,60,66\},\{1,6,11,21,38,50,60,78\}$ $\{1,6,11,21,38,50,73,78\},\{1,8,11,21,33,38,58,70\},\{1,8,11,21,38,55,62,84\}$ $\{1,6,11,21,38,53,62,68\},\{1,6,11,21,38,53,62,82\},\{1,6,11,21,38,53,62,88\}$ $\{1,6,11,21,38,53,68,82\},\{1,6,11,21,38,53,82,89\},\{1,6,11,21,38,54,57,87\}$ $\{1,6,11,21,38,54,68,75\},\{1,6,11,21,38,54,75,87\},\{1,6,11,21,38,57,79,88\}$ $\{1,6,11,21,38,60,73,78\},\{1,6,11,21,38,62,66,82\},\{1,6,11,21,38,62,66,88\}$ $\{1,6,11,21,38,62,68,75\},\{1,6,11,21,38,62,82,89\},\{1,6,11,21,38,73,78,89\}$ $\{1,6,11,21,38,73,82,89\},\{1,8,11,21,30,38,50,70\},\{1,8,11,21,30,38,62,73\}$ $\{1,8,11,21,30,38,70,90\},\{1,8,11,21,30,38,73,78\},\{1,8,11,21,33,38,50,75\}$ $\{1,8,11,21,33,38,58,84\},\{1,8,11,21,33,38,70,79\},\{1,8,11,21,33,38,79,84\}$ $\{1,8,11,21,35,38,55,62\},\{1,8,11,21,35,38,57,86\},\{1,8,11,21,35,38,72,75\}$ $\{1,8,11,21,37,38,50,73\},\{1,8,11,21,37,38,50,77\},\{1,8,11,21,37,38,50,86\}$ $\{1,8,11,21,37,38,55,57\},\{1,8,11,21,37,38,55,76\},\{1,8,11,21,37,38,57,77\}$ $\{1,8,11,21,37,38,77,86\},\{1,8,11,21,38,48,73,90\},\{1,8,11,21,38,48,79,86\}$ $\{1,8,11,21,38,50,70,77\},\{1,8,11,21,38,50,70,90\},\{1,8,11,21,38,55,57,78\}$ $\{1,8,11,21,38,55,58,72\},\{1,8,11,21,38,55,58,76\},\{1,8,11,21,38,55,62,73\}$ $\{1,8,11,21,38,55,73,78\},\{1,8,11,21,38,55,76,84\},\{1,8,11,21,38,57,77,86\}$ $\{1,8,11,21,38,58,70,79\},\{1,8,11,21,38,58,72,90\},\{1,8,11,21,38,58,76,84\}$ $\{1,8,11,21,38,58,76,90\},\{1,8,11,21,38,58,79,84\},\{1,8,11,21,38,58,79,90\}$ $\{1,8,11,21,38,62,76,84\},\{1,8,11,21,38,70,79,90\},\{1,9,11,21,30,38,58,67\}$ $\{1,9,11,21,30,38,59,67\},\{1,9,11,21,30,38,59,82\},\{1,9,11,21,30,38,59,90\}$ $\{1,9,11,21,33,38,55,58\},\{1,9,11,21,33,38,55,64\},\{1,9,11,21,33,38,55,66\}$ $\{1,9,11,21,33,38,58,67\},\{1,9,11,21,33,38,67,78\},\{1,9,11,21,33,38,67,86\}$ $\{1,9,11,21,33,38,70,87\},\{1,9,11,21,36,38,48,70\},\{1,10,11,21,37,38,73,90\}$ $\{1,9,11,21,36,38,48,90\},\{1,9,11,21,36,38,70,82\},\{1,9,11,21,36,38,70,88\}$ $\{1,9,11,21,38,49,58,66\},\{1,9,11,21,38,49,67,75\},\{1,9,11,21,38,49,67,78\}$ $\{1,9,11,21,38,49,67,86\},\{1,9,11,21,38,49,78,87\},\{1,9,11,21,38,55,58,66\}$ $\{1,9,11,21,38,55,62,82\},\{1,9,11,21,38,55,64,88\},\{1,9,11,21,38,55,66,88\}$ $\{1,9,11,21,38,59,67,86\},\{1,9,11,21,38,59,68,82\},\{1,9,11,21,38,59,68,90\}$ $\{1,9,11,21,38,59,75,90\},\{1,9,11,21,38,62,68,90\},\{1,9,11,21,38,62,75,87\}$ $\{1,9,11,21,38,48,59,82\},\{1,9,11,21,38,48,59,86\},\{1,9,11,21,38,48,70,82\}$ $\{1,9,11,21,38,64,70,82\},\{1,9,11,21,38,64,70,88\},\{1,9,11,21,38,64,75,88\}$ $\{1,10,11,21,30,38,53,88\},\{1,10,11,21,34,38,54,68\},\{1,10,11,21,34,38,54,79\}$ $\{1,10,11,21,34,38,64,88\},\{1,10,11,21,34,38,68,86\},\{1,10,11,21,34,38,79,86\}$ $\{1,10,11,21,36,38,54,57\},\{1,10,11,21,36,38,54,76\},\{1,10,11,21,36,38,57,78\}$ $\{1,10,11,21,36,38,59,90\},\{1,10,11,21,36,38,78,84\},\{1,10,11,21,37,38,54,64\}$ $\{1,10,11,21,37,38,54,66\},\{1,10,11,21,37,38,54,90\},\{1,10,11,21,37,38,66,76\}$ $\{1,10,11,21,30,38,49,67\},\{1,10,11,21,30,38,53,67\},\{1,10,11,21,30,38,53,78\}$ $\{1,10,11,21,38,49,66,76\},\{1,10,11,21,38,49,66,79\},\{1,10,11,21,38,49,66,86\}$ 
$\{1,10,11,21,38,49,79,88\},\{1,10,11,21,38,53,57,88\},\{1,10,11,21,38,53,64,67\}$ $\{1,10,11,21,38,53,67,78\},\{1,10,11,21,38,53,78,84\},\{1,10,11,21,38,54,57,79\}$ $\{1,10,11,21,38,54,66,76\},\{1,10,11,21,38,54,79,90\},\{1,10,11,21,38,57,79,88\}$ $\{1,10,11,21,38,59,67,78\},\{1,10,11,21,38,59,79,86\},\{1,10,11,21,38,66,79,86\}$ $\{1,10,11,21,38,67,76,84\},\{1,10,11,21,38,67,78,84\},\{1,10,11,21,38,73,79,90\}$ $\{1,11,21,30,38,49,60,78\},\{1,11,21,30,38,50,59,78\},\{1,11,21,30,38,53,62,90\}$ $\{1,11,21,30,38,53,77,89\},\{1,11,21,35,38,49,62,75\},\{1,11,21,36,38,48,70,90\}$ $\{1,11,21,30,38,54,58,90\},\{1,11,21,30,38,59,77,89\},\{1,11,21,33,38,53,78,89\}$ $\{1,11,21,33,38,54,64,87\},\{1,11,21,33,38,54,75,87\},\{1,11,21,33,38,55,64,84\}$ $\{1,11,21,33,38,55,78,84\},\{1,11,21,33,38,60,68,86\},\{1,11,21,33,38,64,67,75\}$ $\{1,11,21,33,38,64,75,87\},\{1,11,21,33,38,67,75,87\},\{1,11,21,34,38,48,73,82\}$ $\{1,11,21,34,38,49,58,79\},\{1,11,21,34,38,49,62,75\},\{1,11,21,34,38,49,72,88\}$ $\{1,11,21,34,38,49,75,88\},\{1,11,21,34,38,58,79,84\},\{1,11,21,34,38,60,78,84\}$ $\{1,11,21,34,38,72,75,88\},\{1,11,21,34,38,62,75,88\},\{1,11,21,35,38,49,60,76\}$ $\{1,11,21,35,38,49,62,76\},\{1,11,21,35,38,49,75,88\},\{1,11,21,35,38,53,57,67\}$ $\{1,11,21,35,38,53,57,68\},\{1,11,21,35,38,53,57,70\},\{1,11,21,35,38,53,67,76\}$ $\{1,11,21,35,38,53,70,88\},\{1,11,21,35,38,55,59,86\},\{1,11,21,35,38,57,68,86\}$ $\{1,11,21,35,38,57,70,79\},\{1,11,21,35,38,48,59,86\},\{1,11,21,35,38,48,59,89\}$ $\{1,11,21,35,38,59,79,89\},\{1,11,21,35,38,62,68,75\},\{1,11,21,35,38,62,68,76\}$ $\{1,11,21,35,38,62,75,88\},\{1,11,21,35,38,48,67,86\},\{1,11,21,35,38,48,67,89\}$ $\{1,11,21,35,38,48,79,89\},\{1,11,21,36,38,48,60,87\},\{1,11,21,36,38,48,60,90\}$ $\{1,11,21,36,38,48,76,87\},\{1,11,21,36,38,48,76,90\},\{1,11,21,36,38,54,66,76\}$ $\{1,11,21,36,38,55,66,82\},\{1,11,21,36,38,57,72,78\},\{1,11,21,36,38,70,76,90\}$ $\{1,11,21,36,38,72,78,87\},\{1,11,21,37,38,50,64,75\},\{1,11,21,37,38,50,64,77\}$ $\{1,11,21,37,38,55,66,86\},\{1,11,21,37,38,57,73,87\},\{1,11,21,37,38,62,73,89\}$ $\{1,11,21,37,38,64,67,75\},\{1,11,21,37,38,64,75,87\},\{1,11,21,37,38,64,77,87\}$ $\{1,11,21,37,38,66,77,86\},\{1,11,21,37,38,67,75,87\},\{1,11,21,38,48,59,67,86\}$ $\{1,11,21,38,48,59,67,89\},\{1,11,21,38,48,59,82,86\},\{1,11,21,38,48,60,73,87\}$ $\{1,11,21,38,48,60,76,87\},\{1,11,21,38,57,67,78,87\},\{1,11,21,38,57,72,78,87\}$ $\{1,11,21,38,48,60,76,90\},\{1,11,21,38,48,64,70,79\},\{1,11,21,38,49,60,73,87\}$ $\{1,11,21,38,49,67,78,87\},\{1,11,21,38,50,66,77,88\},\{1,11,21,38,50,68,75,90\}$ $\{1,11,21,38,53,57,68,77\},\{1,11,21,38,53,57,70,88\},\{1,11,21,38,53,62,76,84\}$ $\{1,11,21,38,53,64,82,84\},\{1,11,21,38,54,57,72,87\},\{1,11,21,38,54,58,76,90\}$ $\{1,11,21,38,54,59,72,75\},\{1,11,21,38,55,57,73,78\},\{1,11,21,38,55,59,82,86\}$ $\{1,11,21,38,57,73,78,87\}$

$(9,2)-\operatorname{arcs}$ are

$\{1,11,21,30,38,50,70,77,88\},\{1,11,21,30,38,62,73,82,89\},\{1,11,21,33,38,50,60,66,86\}$, $\{1,11,21,33,38,50,60,68,84\},\{1,11,21,34,38,48,64,70,82\},\{1,11,21,34,38,48,64,73,79\}$, $\{1,11,21,36,38,55,72,82,84\},\{1,11,21,37,38,62,66,82,89\},\{1,11,21,38,50,59,72,75,90\}$, $\{1,11,21,38,54,59,68,75,90\},\{1,3,11,21,36,38,55,72,84\}$.

\section{Conclusion}

We had described procedure for searching all complete $(k, 2)-$ arcs with $6 \leq k<10$ containing the quadrangles $O, I, X$, $(a, b, 1)$, with $a \in F_{3}, b \in S \backslash F_{3}$ constructing the Fano subplanes in the left nearfield plane of order 9 . By applying a method and an algorithm (implemented $C \#)$ a full listing the complete $(k, 2)$-arcs were determined and classified . These can be 
represented by four groups. There are 1 classe of the complete $(6,2)-\operatorname{arcs}, 108$ classes of the complete $(7,2)-\operatorname{arcs}, 319$ classes of the complete $(8,2)-\operatorname{arcs}, 11$ classes of the complete $(9,2)-\operatorname{arcs}$.

\section{Competing interests}

The authors declare that they have no competing interests.

\section{Authors' contributions}

All authors have contributed to all parts of the article. All authors read and approved the final manuscript.

\section{References}

[1] Z.Akça - S.Ekmekçi - A.Bayar, On Fano Configurations of the Left Hall Plane of order 9, Konuralp journal of mathematics (to appear) (2016).

[2] E. Altıntaş, On arcs containing the Fano plane in the left near field plane of order 9, ESOGU Sci. Enst, (2015).

[3] M. Hall - Jr, J.D. Swift - R. Killgrove, On projective planes of order nine, Math. Tables and Other Aids Comp. 13 (1959) $233-246$.

[4] T.G. Room - P.B. Kirkpatrick, Miniquaternion Geometry, London, Cambridge University Press, 177, (1971).

[5] M. Taş, On the configuartions of projective plane of order 9 founded over left Hall system, ESOGU Sci. Enst., 2015.

[6] O. Veblen and J.H.M. Wedderburn, Non-Desarguesian and non-Pascalian geometries, Trans. Amer. Math. Soc. 8 (1907), 379-388.

[7] http://fef.ogu.edu.tr/matbil/zakca//FanoPointsFinder. 